\title{
Harnessing Advanced Dynamic Effects for High Performance Directly Modulated Lasers
}

\author{
Zhixin Liu \\ Department of Electronics and Electrical Engineer at University College London, London, WC1E 7JE, UK \\ e-mail address:zhixin.liu@ucl.ac.uk
}

\begin{abstract}
This paper reviews the principle of three dynamic effects, inc. photon-photon resonance, detuned loading, and optical injection locking, for high speed directly modulated lasers. Designs and recent progress are discussed to inspire future research. (C) 2020 The Author(s)
\end{abstract}

\section{Introduction}

Directly modulated lasers (DML) have been used in optical communications ever since the birth of semiconductor lasers. The features of low cost and low power consumption make DML based transceivers promising in cost and size sensitive markets such as data center interconnections, high performance computing and access networks [1]. Comparing to the external modulators (e.g. electroabsorption modulators or Mach-Zehnder modulators), the modulation bandwidth (BW) of conventional solitary DMLs is limited to the range of 15 to $25 \mathrm{GHz}$, making it difficult to support the growing needs for $400 \mathrm{~Gb} / \mathrm{s}$ and $800 \mathrm{~Gb} / \mathrm{s}$ data communications. In addition, the relatively large modulation chirp (typically $10-15 \mathrm{GHz}$ ) broadens the optical spectrum, constraining the transmission distance of high speed signals to $10-40 \mathrm{~km}$ due to the chromatic dispersion (CD) at the low-loss 1550 nm wavelength region in standard single-mode fiber (SMF-28). To enhance the BW, researchers have made numerous efforts engineering semiconductor material for high gain, achieving up to $30 \mathrm{GHz} \mathrm{BW}$ for a solitary laser [2]. Further enhancement of the gain is possible with radically innovations in semiconductor materials such as quantum-dot materials, which has been shown to achieve high gain and small linewidth enhancement factor that significantly reduces modulation chirp [3]. However, the quantum-dot material has an inherently smaller light confinement factor comparing to quantum well materials, which limits the demonstrated BW of QD DML to about $12 \mathrm{GHz}$ [4].

In addition to material engineering, the recent advance of DMLs has been focusing on new laser structures and subsystems that harness laser dynamic effects such as photon-photon resonance (PPR), detuned loading and optical injection locking (OIL). These dynamic effects not only enhance the BW but also reduce the modulation chirp, demonstrating $>50 \mathrm{GH}$ BW that supports $>300 \mathrm{~Gb} / \mathrm{s}$ single-wavelength intensity modulation [5] and has been shown to generate coherent (IQ modulation) signals [6]. In this abstract, I aim to intuitively explain the principle of these dynamic effects and review the recent DMLs development that exploring these dynamic effects.

\section{Dynamic effects and DML designs}

\subsection{Photon-photon Resonance}

Fig.1a shows the structure of the self-feedback laser that consists of a distributed feedback laser (DFB) section and an external cavity with high reflectivity (HR) mirror at the rear side of the laser [7]. The HR mirror reflects the light emitting from the rear facet of the DFB section and re-injects back to the DFB section. This cavity length needs to be carefully designed such that the external cavity creates side modes that locate at the high frequency region of DFB modulation response. When the reflected light is in phase with the DFB section, the photons generated in the high frequency region is resonantly amplified by in the side modes, leading to another resonance in the frequency response which is known as PPR, significantly enhance BW by 2-3 times [7, 8]. As the interaction between the DFB
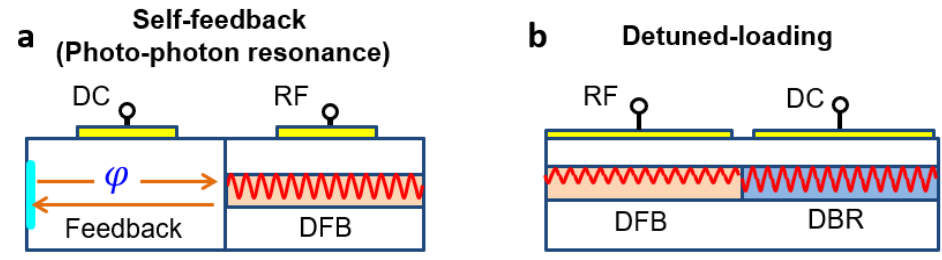
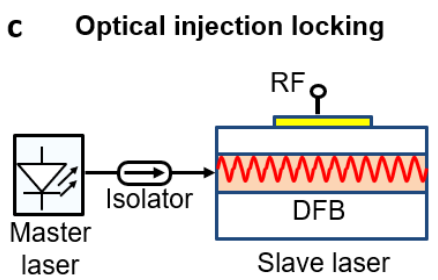

Fig.1 laser and system structures that harness the dynamic effects of (a) PPR, (b) detuned loading, and (c) OIL. 
and external cavity depends on the phase of the reflected light, a DC current is applied to control the phase of the reflected light. Both BW and chirp depend on this phase control. It is possible to simultaneously achieve an enhanced BW with a reduced chirp.

\subsection{Detuned-loading}

Fig. 1b shows the structure of a distributed reflection laser that contains a DFB section and a distributed bragg reflector (DBR) section. Different from the passive feedback laser where the HR mirror that reflects all the wavelength components, the DBR section is a wavelength dependent reflector. When the lasing wavelength of the DFB section locates at the falling edge of the long wavelength side of the DBR, any modulation that increases the injected current will lead to a blue shift of the lasing wavelength towards the reflection peak of the DBR mirror. The increased DBR reflection means a reduced cavity loss that effectively increases the photon density or the effective gain of the DML. This dynamic interaction of cavity loss and lasing wavelength in a DML is known as detuned loading [9], which effectively increases the gain, and correspondingly, the relaxation oscillation frequency and DML BW. Due to the need for an external cavity, the detuned loading effect is always accompanied with PPR effects. The combined effect of detuned loading and PPR has led to a recent demonstration of $65 \mathrm{GHz}$ BW DML with a significantly reduced linewidth enhancement factor of about 0.6 [10]. In addition to the DBR design, the detunedloading effect can also be generated in a " $\mathrm{DFB}+\mathrm{R}$ " structure [11], where a passive waveguide section with a low reflectivity of $\sim 3 \%$ at the front facet is integrated with the DFB section. The low front reflection creates a strong filtering effect in the laser cavity that generates the detuned loading effect.

\subsection{Optical Injection Locking}

OIL refers to a technique that external light is injected into the laser cavity such that the slave laser is frequency and phase synchronized to the injected 'master light' (see Fig.1c) [12]. Different from the self-feedback laser in which the PPR depends on the cavity loss and the reflection phase, the dynamic behavior of an OIL laser depends on the power ratio between the master and slave lasers, known as injection ratio, and the frequency detuning $\left(\Delta f=f_{\text {inj }}-f_{f r e e}\right)$ between the injected master light $\left(\mathrm{f}_{\mathrm{inj}}\right)$ and the free running slave laser $\left(\mathrm{f}_{\text {free }}\right)$. It has been shown that OIL can significantly enhance small-signal modulation BW by more than threefold [13] and suppress modulation chirp, leaving very small residual chirp that depends on the injection ratio (or the locking range). The suppressed chirp offers a unique opportunity of generating arbitrary amplitude-phase modulated signals through coherent synthesis of two or more DMLs $[6,14]$. The main challenge of OIL is how to integrate multiple independent lasers with isolator or circulator in between.

\section{Conclusion}

The advanced dynamic effects reviewed in this abstract have potential to significantly enhance the DML BW with a simultaneous reduction of the modulation chirp. Future DML design should consider integrated solutions to harness one or multiple of these dynamic effects for high performance and low cost optical transmitters.

\section{References}

[1] Y. Matsui, "Directly Modulated Laser Technology: Past, Present, and Future,” (River Publisher 2018), Chap.3.

[2] Y. Matsui et al., "30-GHz bandwidth 1.55- $\mu \mathrm{m}$ strain-compensated InGaAlAs-InGaAsP MQW laser," IEEE PTL, 9, 25-27 (1997).

[3] H. Saito et al., "Low chirp observed in directly modulated quantum dot lasers," IEEE PTL, 12, 1298-1300 (2000).

[4] S. M. Kim et al., "High-Frequency Modulation Characteristics of 1.3- $\mu \mathrm{m}$ InGaAs Quantum Dot Lasers," IEEE PTL, 16, 377-379 (2004).

[5] N.-P. Diamantopoulos et al., "Net 321.24-Gb/s IMDD Transmission Based on a >100-GHz Bandwidth Directly-Modulated Laser," in Proc. OFC, Th4C.1, 2020.

[6] Z. Liu et al., "Modulator-free quadrature amplitude modulation signal synthesis," 5, 5911 (2014)

[7] J. Kreissl et al., "Up to 40-Gb/s Directly Modulated Laser Operating at Low Driving Current: Buried-Heterostructure Passive Feedback Laser (BH-PFL)," IEEE PTL, 24, 362-364 (2011).

[8] Z. Liu et al., "Discrete Multitone Format for Repeater-Less Direct-Modulation Direct-Detection Over 150 km," J. Lightwave Technol. 34, 3223-3229 (2016)

[9] M. Chaciński and R. Schatz, "Impact of losses in the Bragg section on the dynamics of detuned loaded DBR lasers," IEEE J. Quantum Electron, 46,1360-1367 (2010).

[10] Y. Matsui et al., "Isolator-free > 67-GHz bandwidth DFB+R laser with suppressed chirp," in Proc. OFC, paper Th4A.1., 2020

[11] Z. Liu et al., "50-GHz Repetition Gain Switching Using a Cavity-Enhanced DFB Laser Assisted by Optical Injection Locking," J. Lightwave Technol. 38, 1844-1850 (2018)

[12] Z. Liu and R. Slavík, "Optical Injection Locking: From Principle to Applications," J. Lightwave Technol. 38, 43-59 (2020)

[13] E. K. Lau et al., "Enhanced modulation characteristics of optical injection-locked lasers: A tutorial," JSTQE, 15, 618-633 (2009).

[14] Z. Liu et al., "Optical Injection-Locked Directly Modulated Lasers for Dispersion Pre-Compensated Direct-Detection Transmission," J.

Lightwave Technol. 36, 4967-4974 (2018) 